\title{
How the CMB Anisotropy Pattern Could Be a Map of Gravitational Entropy
}

\author{
Eugene Terry Tatum \\ 760 Campbell Ln. Ste. 106 \#161, Bowling Green, KY 42104, USA \\ Email:ett@twc.com
}

How to cite this paper: Tatum, E.T. (2018) How the CMB Anisotropy Pattern Could Be a Map of Gravitational Entropy. Journal of Modern Physics, 9, 1484-1490. https://doi.org/10.4236/jmp.2018.98092

Received: June 12, 2018

Accepted: July 9, 2018

Published: July 12, 2018

Copyright $\odot 2018$ by author and Scientific Research Publishing Inc. This work is licensed under the Creative Commons Attribution International License (CC BY 4.0).

http://creativecommons.org/licenses/by/4.0/

\begin{abstract}
The rationale for Flat Space Cosmology (FSC) calculations of gravitational entropy in the form of $\sqrt{S}$ is presented. These calculations indicate a tight correlation with the COBE DMR measurement showing CMB RMS temperature variations of 18 micro Kelvins. The COBE $d T / T$ anisotropy ratio of 0.66 $\times 10^{-5}$ falls within the FSC gravitational entropy range calculated for the beginning and ending conditions of the recombination/decoupling epoch. Thus, the FSC model incorporating gravity as an emergent property of entropy suggests that the CMB temperature anisotropy pattern could simply be a map of gravitational entropy, as opposed to a magnified "quantum fluctuation" event at a finite beginning of time.
\end{abstract}

\section{Keywords}

Flat Space Cosmology, Cosmic Microwave Background, CMB Anisotropy, Cosmology Theory, Cosmic Entropy, Gravitational Entropy, Black Holes, Standard Cosmology

\section{Introduction and Background}

In the July 2018 issue of Journal of Modern Physics, the paper entitled, "Clues to the Fundamental Nature of Gravity, Dark Energy and Dark Matter," makes a persuasive case in support of gravity being an emergent property of cosmic entropy $S$ [1]. This argument is bolstered by Verlinde's landmark paper on the subject [2] and by Roger Penrose's conception of gravitational entropy [3]. Notably, Penrose's presentation on cosmic entropy, which relies on the Bekenstein-Hawking definition of black hole entropy [4] [5], relates the magnitude of cosmic horizon surface area $\left(4 \pi R^{2}\right)$ with the Lambda term $\Lambda$ in the same way as the Flat Space Cosmology (FSC) model. FSC and Penrose (page 277) have derived $\Lambda$ as always being equal to $3 / R_{t}^{2}$. This, of course, implies that va- 
cuum energy density $\left(\Lambda c^{4} / 8 \pi G\right)$ is not a cosmological constant over the great span of cosmic time, but rather a constantly declining cosmological parameter. This relationship applies only to a general relativity model (such as FSC) of an expanding universe with a finite scaling horizon surface area. Only in a finite universe model can there be a holographic principle.

The key to understanding gravitational entropy, as presented by Penrose (pages 256-258), is that in a gravitating universe, the ongoing clustering of stars and galaxies is in the direction of greater cosmic entropy. Black holes, in particular, are thought to be local reservoirs of maximum entropy. If so, then galactic supermassive black holes must be huge repositories of cosmic entropy. As presented in "Clues to the Fundamental Nature of Gravity, Dark Energy and Dark Matter," gravitational entropy in the form of $\sqrt{S}$ scales in direct proportion to FSC cosmic time, cosmic radius, cosmic matter mass, and cosmic vacuum energy (i.e., dark energy). Cosmic entropy in the correct scale form of $\sqrt{S}$ is always inversely proportional to "Universal Temperature" $T_{u}$, as defined by $T_{u}=T^{2}$, wherein $T^{2}$ is in degrees Kelvin squared. This equal-scaling and proportionality between cosmic gravitational entropy and these FSC parameters allows one to easily calculate the gravitational entropy at any time and temperature in the cosmic past or future. Of particular interest, for the purposes of this paper, is the relative gravitational entropy during the cosmic microwave background (CMB) recombination/decoupling epoch in comparison to the gravitational entropies at one year after the Planck epoch and at current cosmic time in years. Plasma physics and particle physics tells us that the recombination/decoupling event began when our early universe was at about $3000 \mathrm{~K}$. The great preponderance of the $\mathrm{CMB}$ radiation was released during the cosmic time interval extending from when the universe was at $3000 \mathrm{~K}$ to the abrupt "end of decoupling" approximately 115,000 years later [6].

The astute observer will note that there is some difference between the time vs temperature curves used in standard inflationary cosmology as opposed to FSC. This is the subject of the June 2018 Journal of Modern Physics paper entitled, "Temperature Scaling in Flat Space Cosmology in Comparison to Standard Cosmology" [7]. A comparison of these two models in terms of cosmic temperature vs cosmological redshift $z$ is given below. In the FSC model [8] [9] [10], the following formula is used

$$
z \cong\left(\frac{T_{t}^{2}}{T_{o}^{2}}-1\right)^{1 / 2}
$$

wherein $T_{t}$ is the cosmic radiation temperature at any time $t$ and $T_{o}$ is the current observed CMB temperature of $2.72548 \mathrm{~K}$. In standard inflationary cosmology, the following formula is used

$$
T_{C M B} \cong 2.725(1+z)
$$

wherein $T_{C M B}$ represents the $\mathrm{CMB}$ radiation temperature. As derived in the "Temperature Scaling" FSC paper, 


$$
T^{2} t_{y s}=1.085781647371578 \times 10^{11} \mathrm{~K}^{2} \cdot \mathrm{yr} \text { (sidereal years) }
$$

wherein $T^{2}$ is in Kelvin squared units and $t_{y s}$ is cosmic time in sidereal years.

In this context, the current paper analyzes what the FSC model can tell us about the likely effect of gravity on the CMB anisotropy pattern. The implications are discussed in terms of the well-known Sachs-Wolfe effect (See Discussion section below). Particular emphasis is given to the gravitational entropy $\sqrt{S}$ values corresponding to the recombination/decoupling epoch beginning and ending cosmic temperatures. The ensuing discussion will focus on the implications of these gravitational entropy calculations, and what effect gravitational entropy may have had on this CMB anisotropy pattern.

\section{Results}

Equation (3) gives an FSC cosmic time value of about 12,064 years at the beginning of the recombination/decoupling epoch $(3000 \mathrm{~K})$. Thus, for reasons given in the Discussion section, the "end of decoupling" event happened in the FSC model at approximately 127,000 years $(924.63 \mathrm{~K})$ after the Planck epoch. The Planck epoch is the time of the Planck-scale universe and is often considered to be the approximate moment of the "Big Bang" in standard cosmology. The "Clues to the Fundamental Nature of Gravity, Dark Energy and Dark Matter" paper derives

$$
\sqrt{S}=\frac{c \sqrt{\pi}}{L_{p}} t
$$

Showing the direct proportionality relationship between gravitational entropy $\sqrt{S}$ and cosmic time $t$. Speed of light $c$ and Planck length $L_{p}$ are assumed to be constants over cosmic time. Thus, if we operationally define $\sqrt{S}$ in terms of years,

$\sqrt{S}=1$ at 1 year of cosmic time at temperature $3.295 \times 10^{5} \mathrm{~K}$

$\sqrt{S}=12,064$ at 12,064 years of cosmic time at temperature $3000 \mathrm{~K}$

$\sqrt{S}=127,000$ at 127,000 years of cosmic time at temperature $924.63 \mathrm{~K}$

$\sqrt{S}=14.617 \times 10^{9}$ at $14.617 \times 10^{9}$ years of cosmic time at temperature $2.72548 \mathrm{~K}$

The above CMB gravitational entropies $(12,064$ and 127,000) can then be related to current cosmic entropy $\left(14.617 \times 10^{9}\right)$ as follows:

[ $\sqrt{S}$ at the beginning of $\mathrm{CMB}$ emission $] /[\sqrt{S}$ at current time $]=8.25 \times 10^{-7}$ $\left(0.825 \times 10^{-6}\right)$

[ $\sqrt{S}$ at the ending of $\mathrm{CMB}$ emission] $/[\sqrt{S}$ at current time $]=8.69 \times 10^{-6}$ $\left(0.869 \times 10^{-5}\right)$

\section{Discussion}

Sachs and Wolfe [11], using a gravitational redshift theoretical argument, suggested that $\mathrm{CMB}$ temperature anisotropy could be a result of inhomogeneous gravitational particle clustering already present at the time of recombina- 
tion/decoupling. Their 1967 gravitational redshift argument for what is known as "the Sachs-Wolfe effect" is now widely believed to be correct [12]. The Sachs-Wolfe effect is widely considered to be the source of large angular scale temperature fluctuations in the CMB.

However, in a spatially flat universe, the Sachs-Wolfe effect can also be considered to be the source of the smaller angular scale fluctuations of the CMB temperature anisotropy [13]. The Boomerang Collaboration [14] reported CMB anisotropy observations closely fitting "the theoretical predictions for a spatially flat cosmological model with an exactly scale invariant primordial power spectrum for the adiabatic growing mode" [Bucher (2015), page 6]. The Boomerang, Wilkinson Microwave Anisotropy Probe (WMAP) [15], and Planck satellite [16] $\mathrm{CMB}$ anisotropy studies have all confirmed global spatial flatness of the universe at the time of the recombination/decoupling epoch. Therefore, in terms of the "gravitational potential variations" explanation first proposed by Sachs and Wolfe, little in the theory of CMB temperature anisotropy has changed since 1967. What has changed since the time of the Sachs and Wolfe paper is the precision of measurements of the CMB temperature anisotropy. Both the WMAP study and the more sensitive Planck study have confirmed the CMB temperature anisotropy to be on the order of approximately one part per 100,000 $\left(10^{-5}\right)$.

At the time of these CMB study reports, the extreme flatness observations of the $\mathrm{CMB}$ temperature anisotropy were credited as a victory for cosmic inflation. However, there was no basis to determine which particular theoretical version of inflation was correct, or even whether another flat space cosmology theory without an inflationary mechanism (such as FSC) could, in fact, be an even better explanation of global cosmic flatness observations. The following quote from physicist Philip Gibbs sums it up best: "The problem... is that no particular model of inflation has been shown to work yet. It is possible that work has not yet been completed or that a more recent specific model will be shown to be right" [17] [18]. In a soon-to-be-published FSC paper, this author will clearly show why the FSC model is superior to the standard inflationary model, using a series of specific FSC model predictions dating back to 2015.

As mentioned in the Introduction and Background section, current best estimates of the cosmic time interval during which the $\mathrm{CMB}$ radiation was released suggest that the recombination/decoupling epoch lasted approximately 115,000 years. In standard cosmology this is believed to have occurred between approximately 372,000 and 487,000 years after a "Big Bang" at or near the Planck epoch. In the FSC model, the temperature scaling is slightly different [Tatum, et al (2018)], placing the beginning of the recombination/decoupling epoch (3000 K) at approximately 12,064 years after the Planck epoch. Adding the estimated time interval of approximately 115,000 years puts the FSC "end of decoupling" event at about 127,000 years after the Planck epoch.

Gravitational entropy $\sqrt{S}$ in the FSC model follows the same log value scale as cosmic time. Thus, there should be a uniform progression from maximum gravitational potential "smoothness," corresponding to any operationally-defined 
"minimal" or "beginning" anisotropy, to ongoing and progressively greater gravitational inhomogeneity (i.e., "filaments", "clusters" and "voids"). Furthermore, this is consistent with the concept that cosmic entropy smoothly increases as the expanding cosmic horizon surface area (the Bekenstein-Hawking measure of entropy) increases. Thus, it would seem reasonable to assume that, if the $C M B$ temperature anisotropy pattern is in keeping with the Sachs-Wolfe effect for a spatially flat universe, and if gravity is truly an emergent property of cosmic entropy as indicated by Verlinde, the FSC gravitational entropy values pertaining to the recombination/decoupling epoch should also be a measure of the CMB temperature anisotropy.

The COBE DMR experiment measured CMB RMS temperature variations of 18 micro Kelvins $\left(1.8 \times 10^{-5} \mathrm{~K}\right)[19]$. This gives a $d T / T$ anisotropy ratio of $(0.000018) / 2.725$, equaling $6.6 \times 10^{-6}$ or $0.66 \times 10^{-5}$. Little has changed in this respect, judging from the subsequent WMAP and Planck CMB temperature anisotropy findings (also approximately $10^{-5}$ ).

It is intriguing that the FSC gravitational entropy ratios provided and calculated at the end of the Results section are $0.825 \times 10^{-6}$ at the beginning of recombination/decoupling and $0.869 \times 10^{-5}$ at the "end of decoupling." It should be noted that the "last scattering surface" is actually a 115,000 year thick segment of microwave radiation spectrum rather than an infinitely thin "surface" at a single redshift. In this context, the COBE DMR $d T / T$ anisotropy ratio of $0.66 \times 10^{-5}$ can only be, in some way, an averaging of the actual ratio numbers pertaining to the beginning and ending conditions responsible for the "last scattering surface." Therefore, the FSC model incorporating gravity as an emergent property of entropy suggests that the $\mathrm{CMB}$ temperature anisotropy pattern could simply be a map of gravitational entropy, as opposed to a magnified "quantum fluctuation" event at a finite beginning of time.

\section{Summary and Conclusions}

The purpose of this paper has been to show how the CMB temperature anisotropy pattern could be a map of gravitational entropy as defined by Roger Penrose in his book entitled, "Fashion, Faith and Fantasy in the New Physics of the Universe." This is particularly relevant with respect to Erik Verlinde's theory that gravity is an emergent property of cosmic entropy. Verlinde's theory dovetails nicely with the July 2018 Journal of Modern Physics paper entitled, "Clues to the Fundamental Nature of Gravity, Dark Energy and Dark Matter."

In the present paper, the rationale for FSC calculations of gravitational entropy in the form of $\sqrt{S}$ is presented. These calculations indicate a tight correlation with the COBE DMR measurement showing CMB RMS temperature variations of 18 micro Kelvins. The COBE $d T / T$ anisotropy ratio of $0.66 \times 10^{-5}$ falls within the FSC gravitational entropy range calculated for the beginning and ending conditions of the recombination/decoupling epoch. Thus, the FSC model incorporating gravity as an emergent property of entropy suggests that the $\mathrm{CMB}$ 
temperature anisotropy pattern could simply be a map of gravitational entropy, as opposed to a magnified "quantum fluctuation" event at a finite beginning of time.

\section{Dedications and Acknowledgements}

This paper is dedicated to Dr. Stephen Hawking and Dr. Roger Penrose for their groundbreaking work on black holes and their possible application to cosmology. Dr. Tatum also thanks Dr. Rudolph Schild of the Harvard Center for Astrophysics for his past support and encouragement.

\section{References}

[1] Tatum, E.T. and Seshavatharam, U.V.S. (2018) Journal of Modern Physics, 9, 1469-1483. https://doi.org/10.4236/jmp.2018.98091

[2] Verlinde, E. (2010) On the Origin of Gravity and the Laws of Newton. arXiv:1001.0785v1 [hep-th].

[3] Penrose, R. (2016) Fashion Faith and Fantasy in the New Physics of the Universe. Princeton University Press, Princeton, US. https://doi.org/10.1515/9781400880287

[4] Bekenstein, J.D. (1974) Physical Review D, 9, 3292-3300. https://doi.org/10.1103/PhysRevD.9.3292

[5] Hawking, S. (1976) Physical Review D, 13, 191-197. https://doi.org/10.1103/PhysRevD.13.191

[6] Spergel, D.N., et al. (2003) Astrophysical Journal Supplement, 148, 175-194. arXiv:astro-ph/0302209. https://doi.org/10.1086/377226

[7] Tatum, E.T. and Seshavatharam, U.V.S. (2018) Journal of Modern Physics, 9, 1404-1414. https://doi.org/10.4236/jmp.2018.97085

[8] Tatum, E.T., Seshavatharam, U.V.S. and Lakshminarayana, S. (2015) International Journal of Astronomy and Astrophysics, 5, 116-124. https://doi.org/10.4236/ijaa.2015.52015

[9] Tatum, E.T., Seshavatharam, U.V.S. and Lakshminarayana, S. (2015) Journal of Applied Physical Science International, 4, 18-26.

[10] Tatum, E.T., Seshavatharam, U.V.S. and Lakshminarayana, S. (2015) Frontiers of Astronomy, Astrophysics and Cosmology, 1, 98-104. http://pubs.sciepub.com/faac/1/2/3

[11] Sachs, R.K. and Wolfe, A.M. (1967) Astrophysical Journal, 147, 73-90. https://doi.org/10.1086/148982

[12] Wright, E.L. (2003) Theoretical Overview of Cosmic Microwave Background Anisotropy. In: Freedman, W.L., Ed., Measuring and Modeling the Universe, Cambridge University Press, Cambridge, 291.

[13] Bucher, M. (2015) Physics of the Cosmic Microwave Background Anisotropy. arXiv:1501.04288v1 [astro-ph.CO]. https://doi.org/10.1142/S0218271815300049

[14] De Bernardis, P., et al. (2000) A Flat Universe from High-Resolution Maps of the Cosmic Microwave Background Radiation. arXiv:astro-ph/0004404v1. https://doi.org/10.1038/35010035

[15] Bennett, C.L. (2013) Nine-Year Wilkinson Microwave Anisotropy Probe (WMAP) Observations: Final Maps and Results. arXiv:1212.5225v3 [astro-ph.CO]. 
https://doi.org/10.1088/0067-0049/208/2/20

[16] Planck Collaboration (2014) Astronomy \& Astrophysics, 23, 1-48.

[17] Keating, B. (2018) Losing the Nobel Prize. W. W. Norton \& Company, New York.

[18] Gibbs, P.E. (2014) Prespacetime Journal, 5, 230-233.

https://prespacetime.com/index.php/pst/article/download/614/612

[19] Wright, E.L., et al. (1996) Astrophysical Journal, 464, L21-L24.

https://doi.org/10.1086/310073 